\title{
Variable Dislocation Widths in Colloidal Crystals of Soft Thermosensitive Spheres
}

\author{
J. Hilhorst and A. V. Petukhov* \\ Van 't Hoff Laboratory for Physical and Colloid Chemistry, Debye Institute for NanoMaterials Science, Utrecht University, \\ Padualaan 8, 3584 CH Utrecht, The Netherlands \\ (Received 15 April 2011; published 25 August 2011)
}

\begin{abstract}
We report on detailed measurements of the core structure of Shockley partial dislocations in colloidal crystals. In crystalline arrays of micrometer sized thermosensitive particles, the interactions between the colloidal building blocks were tuned by changing the temperature. Individual dislocation cores were observed in a confocal microscope and their behavior as a function of temperature was studied. The obtained results qualitatively agree with the Peierls theory and are promising for further studies in which both Peierls stress and dislocation core width are measured simultaneously.
\end{abstract}

PACS numbers: 61.72.Ff, 07.60.Pb, 61.72.Lk, 82.70.Dd

For over a century, since the early experiments on colloids by Perrin [1], colloidal particles have been employed as a model system for atoms. The merits of using colloids as model atoms are reflected in a process such as crystal nucleation. Nucleation of an atomic crystal occurs very quickly and on a length scale of angstroms or nanometers, precluding a real space study of crystal nucleation. The same process in a colloidal system takes minutes and can be observed directly with a microscope [2], while the physics behind the process remains essentially the same. In this way, homogeneous nucleation [2], as well as nucleation at impurity dopants [3], has been studied in great detail. In many ways, this example illustrates one of the key aspects of colloid science: to generate slow and seeable model systems.

Another area of research that could benefit from the colloidal approach is dislocation theory. The historic Peierls theory for dislocations was put forward shortly before the outbreak of World War II [4]. Over a decade later, the core structure of dislocations was observed experimentally for the first time, as a result of the advent of high resolution electron microscopy [5-7]. Despite these advances a direct comparison between core structure and macroscopic material properties has not been made. Here, we will discuss a system of colloidal thermosensitive spheres that readily crystallize into a random hexagonal close packed lattice. Through analysis of confocal microscopic images, Shockley partial dislocations can be identified and characterized. The width of the dislocations is shown to be strongly dependent on temperature, which is discussed within the light of the generalized Peierls theory [8] and the properties of the thermosensitive colloids. The results hold promise for experiments in which dislocation width and crystal shear strength are measured simultaneously.

Colloidal crystals form an ideal system to study dislocations. Because of the weak interactions between the particles, the formation of dislocations costs relatively little energy. In addition, because of the extremely low stacking fault energy, Shockley partial dislocations can exist in unpaired form, i.e., without the presence of a second Shockley partial with Burgers vectors adding up to a full dislocation [9]. Other peculiar dislocations have been observed, such as discrete Shockley partials and partial dislocation loops [10]. The size of the colloids allows for an accurate microscopic characterization of a crystallite, where particle coordinates can be determined in 3D through the use of image analysis software [11].

The colloidal system we employed consists of spheres made of a copolymer of $\mathrm{N}$-isopropylacrylamide (NIPAm) and acrylic acid (AAc) monomers, cross-linked with $N$, $N^{\prime}$-methylenebisacrylamide (BIS) and dyed with 4-methylaminoethylmethacrylate-7-nitrobenzo-2-oxa-1,3-diazol (NBD-MAEM) for confocal microscopy. PolyNIPAm spheres are known for their strong temperature dependent behavior [12], with their diameter decreasing up to a factor of 3 upon heating them above the lower critical solution temperature (LCST) at $32{ }^{\circ} \mathrm{C}[12]$. Below this temperature, the polymer has a hydrophilic character and is swollen with water. Above it, hydrophobic interactions dominate; most of the water is expelled and the particle shrinks [13]. This property has enabled studies of annealing and controlled melting $[14,15]$ of colloidal crystals through control over the particle volume fraction. The added acrylic acid monomer adds $p \mathrm{H}$-dependent size behavior to the spheres, as well as additional charge stabilization at high temperature and $p \mathrm{H}[16]$. We have used particles containing $11 \mathrm{~mol} \%$ of acrylic acid at $p \mathrm{H} 8$. In addition to high stability at temperatures above the LCST, these particles have reduced thermosensitive behavior, enabling more accurate control over the particle volume fraction. The particles were crystallized in flat capillaries consisting of two microscope slides glued on top of each other with two small glass slides as spacer in between. Particle concentration was $2.2 \mathrm{wt} \%$, a concentration that was empirically found to produce large crystals throughout the sample. Measurements were performed in a Linkam THMS600 stage mounted on top of a Nikon TE2000 inverted 

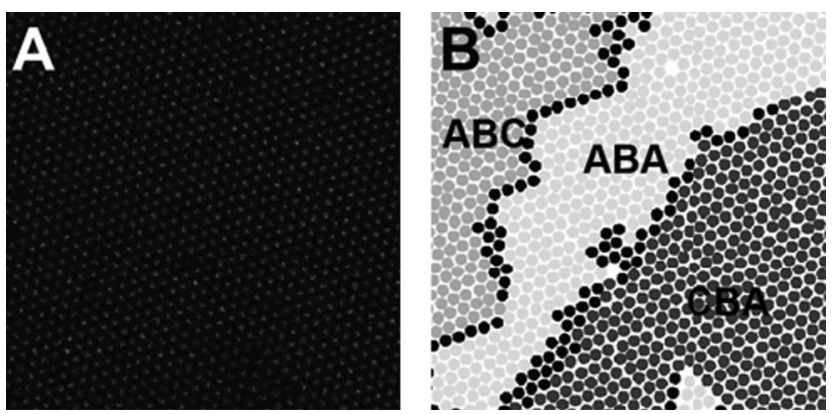

FIG. 1. (a) A typical confocal micrograph of a crystal of polyNIPAm spheres. (b) Schematic representation of the crystal structure of the same crystal layer as in (a). Different shades of gray represent different crystal structures. Black particles have no identifiable crystal structure, as they are located in dislocation cores. Note that for the distinction between crystal structures the particle positions in the layers above and below the characterized layer are used as well.

microscope with a $\mathrm{C} 1$ confocal scan head. Crystals were imaged layer by layer at temperatures ranging from $20^{\circ} \mathrm{C}$ to $40^{\circ} \mathrm{C}$. Above that, dye fluorescence was quenched too much to obtain reliable particle coordinates.

Figure 1(a) shows a typical micrograph of a single layer. After finding particle coordinates using an algorithm in interactive data language [11], the crystalline environment of all particles was determined as described before [10], resulting in images like Fig. 1(b). The different shades of gray distinguish two crystal phases: hexagonal close packed (hcp) or face centered cubic (fcc), with different stacking directions ( $A B A$ vs $B A B$ or $A B C$ vs $C B A$ ). When a transition between two phases is found, a partial dislocation must be present. This can be confirmed by plotting the coordinates of the two layers containing the dislocation on top of each other [Figs. 2(a)-2(c)]. Here, the lattice deformation around the dislocation core is clearly visible. We found that dislocation lines most often run along the $\langle 112\rangle$ or $\langle 110\rangle$ directions and that in both cases dislocations with a dominating screw component were by far the most abundant, as expected from their lower line energy [17].

Using the hexagonal order parameter $\psi_{6}$, the extent of the deformation in the dislocation core region can be accurately determined [9]. The $\psi_{6}$ order parameter, defined as

$$
\psi_{6, j}=\frac{1}{N} \sum_{k=1}^{N} e^{6 i \theta_{j k}}
$$

for each particle $j$, is a measure for the hexagonal order of a particle. It is a complex sum over all $N$ in-plane nearest neighbors of a particle, where $\theta_{j k}$ is the angle between a fixed reference axis and the vector between particle $j$ and neighbor particle $k$. The absolute value of this parameter is an indication of the (hexagonal) crystallinity of the particle within a layer, and its complex argument indicates the local orientation of the lattice with respect to the reference axis. Around a dislocation, the lattice is slightly deformed, resulting in a different orientation of the lattice.

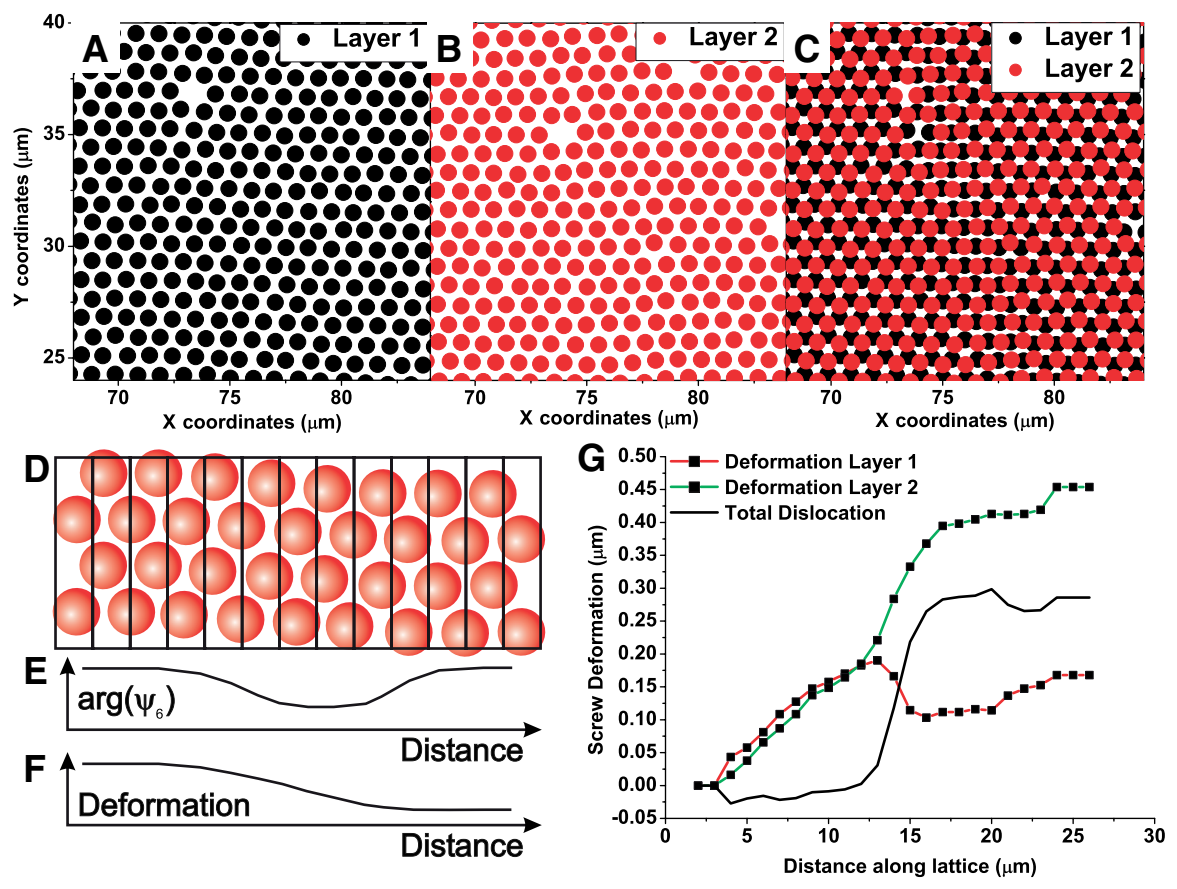

FIG. 2 (color online). Panels (a)-(c) show two subsequent hexagonal layers containing a dislocation. The individual layers in (a) and (b) look normal, but their overlay in (c) shows a dislocation running from top to bottom at $x \approx 76 \mu \mathrm{m}$. In (d) the lines are shown that divide a layer into bins. For each bin, the lattice orientation is determined, resulting in a plot like (e). The corresponding lattice deformation is given in ( $\mathrm{f}$ ). Panel (g) shows the lattice deformations for two individual layers and their difference: a dislocation. 
To quantify the extent of the deformation, the argument of $\psi_{6}$ is averaged over several particles in a small bin parallel to the dislocation line [Figs. 2(d) and 2(e)]. The tangent of this angle multiplied with the width of the bin gives the deformation in the direction along the dislocation line, i.e., the screw component of the dislocation. The sum of the deformations in all bins provides a detailed curve of the total screw component of the dislocation in a single layer [Fig. 2(f)]. Since the dislocation is contained between two layers, both layers have to be characterized and the difference taken to result in the final dislocation curve [Fig. 2(g)]. The resulting curve can be fitted to an arctangent function of the form

$$
u(x)=\frac{b}{\pi} \arctan \frac{x}{w}-\frac{b}{2},
$$

which corresponds to the original solution that was obtained by Peierls for a dislocation profile [4] and is still used in more complex solids [8]. Such a fit yields the Burgers vector $b$ and the dislocation width $w$, which is a measure for the range over which the dislocation is spread. This is a measure for the plastic shear strength of a crystal according to

$$
\sigma_{P}=\frac{\mu b}{a} e^{-2 \pi w / a}
$$

where $\mu$ is the bulk shear modulus of the material and $a$ is the lattice constant perpendicular to the dislocation line.

Using the $\psi_{6}$ method, we studied the behavior of unpaired $30^{\circ}$ Shockley partials with dislocation lines along $\langle 110\rangle$ as a function of temperature. The configuration of such a dislocation is shown in Fig. 3(a). It is a dislocation of mixed type with a dominating screw character. The widths of the dislocations, as obtained from arctangent fits of the deformation profiles, are plotted as a function of temperature in Fig. 3(b). It is evident that the dislocation width is strongly temperature dependent, decreasing by a factor of 4 between $20^{\circ} \mathrm{C}$ and $40^{\circ} \mathrm{C}$. According to Eq. (3), this implies that the Peierls stress strongly increases and the crystal "hardens." This may seem counterintuitive at first, since crystals of polyNIPAm particles have been shown to melt upon heating in earlier studies [14]. However, the added acrylic acid comonomers reduce the thermal response of the particles in such a way that their reaction to temperature change is not strong enough to melt the crystal. In fact, in our measurements, due to slow heating and sample equilibration, the particle number density increased under the influence of gravity upon heating, keeping particle volume fraction approximately constant over the whole temperature range. The observed decrease of dislocation width and consequent crystal hardening is therefore not caused by a gradual transition towards the melting point, but has to be due to a change in the interaction potential between the particles. As the temperature is increased and particles shrink, their repulsive potential hardens [18]. This is commonly described by a potential of the form $U=\varepsilon\left(\frac{\sigma}{r}\right)^{n}$, where $\sigma$ represents the particle diameter, $r$ is the interparticle distance, and $\varepsilon$ is a measure for the strength of the repulsion. The factor $n$ in the exponent determines the "hardness" of the potential. If $n$ goes to infinity, the particles behave like hard spheres, while for $n=1$ the potential is Coulomb-like. For polyNIPAm particles, the value of $n$ has been shown to range from 12 at low temperature to hard-sphere-like at high temperatures [18]. Even though our particles do not shrink dramatically, the value of $n$ is still increasing with temperature.

A recent study by Tretiakov and Wojciechowski [19] shows that the value of $n$ strongly influences the crystal Poisson's ratio, a parameter that has been known to affect dislocation widths since Peierls' early calculations. As this influence strongly depends on the crystal structure and dislocation orientation, we have calculated the dependence of dislocation width on Poisson ratio for this particular
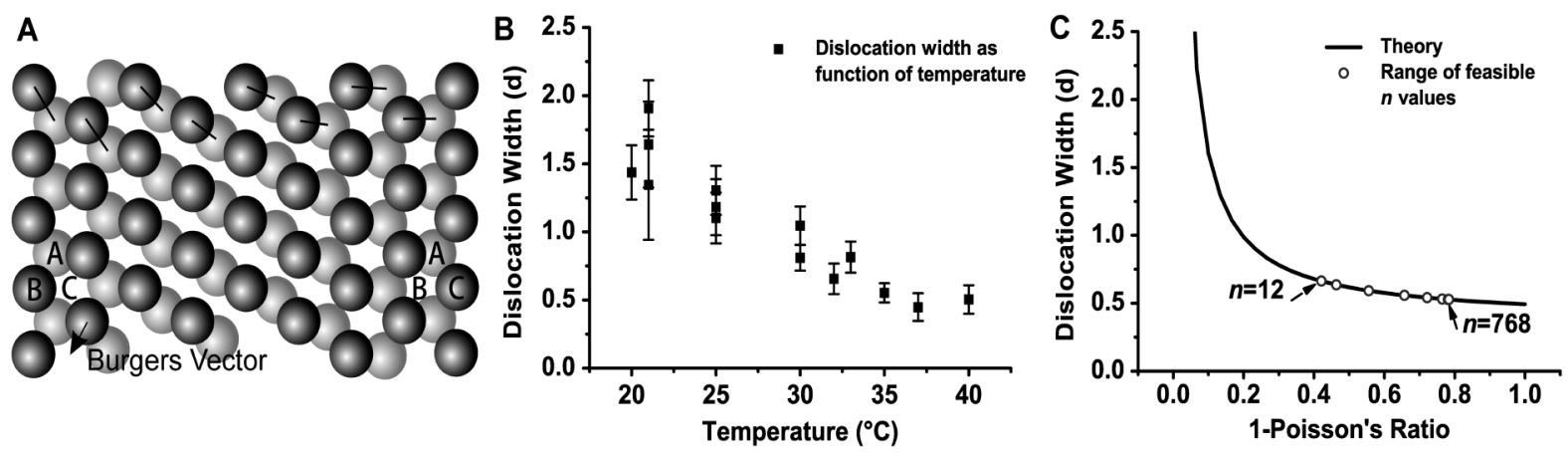

FIG. 3. (a) Representation of the $30^{\circ}$ Shockley partial that was studied. The dislocation line lies vertical in this image, along the $\langle 110\rangle$ direction. The Burgers vector is shown in the bottom left and has a $30^{\circ}$ angle with respect to the dislocation line. The lines connecting the particles in the top of the figure illustrate the gradual deformation of the lattice as particles move from a $B$ to a $C$ position. (b) Plot of the measured dislocation widths as a function of temperature. (c) Calculated dislocation widths as a function of Poisson's ratio. The empty circles represent dislocation widths that can be obtained for soft spheres using the elastic constants calculated by Tretiakov et al. [19]. 
case, following the procedure in Ref. [8]. In this reference, influences of the width of partial dislocations on the dislocation energy are discussed for a dissociated screw dislocation along the $\langle 110\rangle$ direction in a fcc $\{111\}$ plane, within a Peierls framework. The basis of this framework is that the energy of a dislocation consists of an elastic contribution and a misfit energy. The elastic part results from the compression or dilation of the lattice around a dislocation. Elastic energy is minimized by spreading the deformations as far as possible, increasing dislocation width. The misfit energy, caused by nonideal alignment of particles across the glide plane, is minimized by localizing the deformations. Therefore, for the arctangent profiles used here, $w$ is large for dominating elastic interactions and small for dominating misfit energy.

Assuming such arctangent deformations for the edge and screw parts of a dissociated $\langle 110\rangle$ screw dislocation, elastic theory can be applied to obtain an expression for the elastic energy as function of the lattice deformations. For a dissociated screw dislocation along $\langle 110\rangle$ in a fcc lattice, the solution is given by [8]

$$
\begin{aligned}
E_{\mathrm{el}}= & \frac{\mu b^{2}}{4 \pi}\left\{\ln \left[\frac{R}{2 w}\right]-\frac{1}{4} \ln \left[\left(\frac{d}{2 w}\right)^{2}+1\right]\right. \\
& \left.+\frac{1}{12(1-\nu)} \ln \left[\left(\frac{d}{2 w}\right)^{2}+1\right]\right\} .
\end{aligned}
$$

Here, $\mu$ is the $\{111\}$ plane shear modulus, $R$ the dislocation cutoff radius, $\nu$ the Poisson ratio, and $d$ the separation distance between partial dislocations.

Looking more closely at the various terms in Eq. (4), it can be noted that the first term corresponds to the elastic energy of a full screw dislocation, while the second and third terms arise from the splitting of the dislocation in two Shockley partials.

As the partial dislocations studied here were present in unpaired form due to the low stacking fault energy, $d$ is much larger than $w$. In this particular case, if the energy of the stacking fault between the two partials is assumed to be negligible, the misfit energy $E_{A}$ depends linearly on dislocation width:

$$
E_{A}=\frac{\sqrt{3} \mu b^{2}}{2 \pi^{2} c} w,
$$

where $c$ is the $\{111\}$ interlayer spacing. This expression results from an integration of the misfit potential over the whole dislocation [8]. The stable dislocation width is located at the minimum of the sum of elastic and misfit contributions. This can be found by setting the derivative with respect to $w$ of the total energy, $E_{A}+E_{\mathrm{el}}$, to zero:

$$
\begin{aligned}
\frac{\partial E}{\partial w}= & \frac{\mu b^{2}}{4 \pi}\left\{\frac{-1}{w}+\frac{1}{2} \frac{d^{2}}{4 w^{2}+d^{2} w}\right. \\
& \left.-\frac{1}{6(1-\nu)} \frac{d^{2}}{4 w^{2}+d^{2} w}+\frac{2 \sqrt{3}}{\pi c}\right\}=0 .
\end{aligned}
$$

For $d \gg w$, this leads to

$$
w=\frac{\pi c}{2 \sqrt{3}}\left(\frac{1}{2}+\frac{1}{6(1-\nu)}\right),
$$

with Poisson's ratio as the only variable.

A plot of dislocation widths as a function of Poisson's ratio is shown in Fig. 3(c)(note that the $x$ axis displays 1 minus Poisson's ratio). Comparing this image with Fig. 3(b), it can be seen that the full range of measured dislocation widths can be covered by changing Poisson's ratio between 0 and 1 . Although values of Poisson's ratio above 0.5 are impossible for isotropic materials, in anisotropic materials Poisson's ratio can assume arbitrary values for specific directions [19]. Using the values of the elastic constants presented by Tretiakov and Wojciechowski [19] for fcc crystals of soft spheres, Poisson's ratio for compression along $\langle 112\rangle$ could be calculated as a function of particle softness (details of the calculations are given in the Supplemental Material [20]). This allowed for a direct comparison between dislocation width and particle softness. Figure 3(c) displays the range of calculated dislocation widths obtained for particles with values of the softness parameter $n$ ranging from 12 to 768, i.e., from fully swollen polyNIPAm particles to almost hard spheres.

Dislocation widths ranging from 0.78 particle diameters for soft spheres $(n=12)$ to 0.42 particle diameters for hard spheres $(n=768)$ were obtained from the calculations. The trend follows the measured results qualitatively; as temperature increases, particle repulsive potentials become steeper (larger $n$ ), reducing Poisson's ratio as shown in Fig. 3(c), thereby reducing dislocation width. This shows that standard dislocation theory is suitable for qualitatively describing dislocation behavior as a function of temperature in soft-sphere crystals.

Quantitatively, there is a discrepancy between measurements and calculations, mainly at low temperature. Considering the assumptions that have been made in the model, the discrepancy is small, but some improvements may yield even better agreement. On the one hand, the trial functions for the dislocation profile may be too simple, and on the other hand, more complex models should be used for calculations of the elastic and misfit energies. Both possibilities have been discussed by Schoeck [8]. An absolute comparison between theory and experiment, therefore, requires extension of the currently available theoretic framework. Efforts in this direction may benefit from further measurements on colloidal systems, for example, on the effects of dislocation orientation on its width. Additionally, state-of-the-art microscopy setups, combined with shear cells [21] or optical tweezers [22], may be employed to directly measure the Peierls stress, while simultaneously measuring dislocation widths, allowing yet another direct comparison between Peierls theory and experiment.

In conclusion, we have presented direct measurements of dislocation widths as function of temperature in softsphere colloidal crystals. The observed trend can be 
explained qualitatively within a Peierls framework applied to hexagonal crystals. The trend is found to be due to a change in particle interaction potential, leading to a decrease in Poisson ratio with temperature. Quantitatively, the range of measured widths exceeds the predicted values by a factor of $\sim 2-3$, indicating the necessity for further theoretical efforts to close this gap. Despite this absolute discrepancy, results are promising for further experiments where Peierls stress and dislocation width are measured simultaneously.

We thank V.W. A. de Villeneuve and G. Schoeck for useful discussions.

*Corresponding author. a.v.petukhov@uu.nl

[1] J. Perrin, Ann. Chim. Phys. 18, 5 (1909).

[2] U. Gasser, E. R. Weeks, A. Schofield, P. N. Pusey, and D. A. Weitz, Science 292, 258 (2001).

[3] V. W. A. de Villeneuve, R. P. A. Dullens, D. G. A. L. Aarts, E. Groeneveld, J. H. Scherff, W. K. Kegel, and H. N. W. Lekkerkerker, Science 309, 1231 (2005).

[4] R. Peierls, Proc. Phys. Soc. London 52, 34 (1940).

[5] W. Bollmann, Phys. Rev. 103, 1588 (1956).

[6] J. W. Menter, Proc. R. Soc. A 236, 119 (1956).

[7] P. B. Hirsch, R. W. Horne, and M. J. Whelan, Philos. Mag. 1, 677 (1956).

[8] G. Schoeck, Philos. Mag. A 69, 1085 (1994).
[9] V.W.A. De Villeneuve, Structure and Dynamics at Colloidal Boundaries (Utrecht University, Utrecht, 2008).

[10] J. M. Meijer, V. W. A. De Villeneuve, and A. V. Petukhov, Langmuir 23, 3554 (2007).

[11] J. C. Crocker and D. G. Grier, J. Colloid Interface Sci. 179, 298 (1996).

[12] R. Pelton, Adv. Colloid Interface Sci. 85, 1 (2000).

[13] R. Pelton, J. Colloid Interface Sci. 348, 673 (2010).

[14] A. M. Alsayed, M. F. Islam, J. Zhang, P. J. Collings, and A. G. Yodh, Science 309, 1207 (2005).

[15] Y. Peng, Z. Wang, A. M. Alsayed, A. G. Yodh, and Y. Han, Phys. Rev. Lett. 104, 205703 (2010).

[16] M. Karg, I. Pastoriza-Santos, B. Rodriguez-Gonzalez, R. von Klitzing, S. Wellert, and T. Hellweg, Langmuir 24, 6300 (2008).

[17] D. Hull and D.J. Bacon, Introduction to Dislocations (Elsevier, Oxford, 2001), 4th ed., p. 70.

[18] D. M. Heyes and A.C. Branka, Soft Matter 5, 2681 (2009).

[19] K. V. Tretiakov and K. W. Wojciechowski, J. Phys. Chem. B 112, 1699 (2008).

[20] See Supplemental Material at http://link.aps.org/ supplemental/10.1103/PhysRevLett.107.095501 for a detailed overview of the calculations of the $\langle 112\rangle$ Poisson's ratio.

[21] D. Derks, H. Wisman, A. van Blaaderen, and A. Imhof, J. Phys. Condens. Matter 16, S3917 (2004).

[22] M. Yada, J. Yamamoto, and H. Yokoyama, Phys. Rev. Lett. 92, 185501 (2004). 